\title{
Dimensionamento de sistema de microirrigação através de programação não linear considerando-se diferentes demandas hídricas
}

\author{
Kennedy F. M. Lucena' ${ }^{1}$, Carlos de O. Galvão ${ }^{2}$ \& Heber P. Gomes ${ }^{3}$
}

\begin{abstract}
RESUMO
Neste trabalho é analisado o dimensionamento ótimo de sistemas de microaspersão, através de um modelo de otimização utilizando-se programação não linear, com o objetivo de minimizar os custos de investimento e operacional, para três cenários de demandas hídricas obtidas em duas localidades da Região Nordeste do Brasil: Iguatu, CE, com os balanços hídricos anual e mensal, e João Pessoa, PB, com balanço hídrico mensal. O modelo tem como variáveis de decisão: os comprimentos dos trechos de tubos com seus respectivos diâmetros, o tempo total diário de irrigação, a uniformidade de emissão, a freqüência de irrigação e a pressão média de operação do emissor. Os resultados evidenciaram que em regiões de clima semi-árido, como Iguatu, não há diferenças importantes no dimensionamento do sistema com as demandas obtidas através do balanço hídrico anual ou mensal. O custo de investimento não foi afetado pelas demandas, mas apenas os custos operacional e total do sistema. Regiões com menor requerimento hídrico diário e anual, como João Pessoa, permitem maior número de unidades operacionais do sistema e, portanto, maior redução dos custos de investimento, porém para um mesmo número de subunidades e de unidades operacionais as diferenças de demandas hídricas não afetam o dimensionamento. A máxima demanda hídrica diária da cultura tem maior efeito sobre a hidráulica do sistema do que a demanda hídrica anual. O sistema com dimensionamento ótimo depende da análise conjunta do número de subunidades e de unidades operacionais do sistema e não se pode desprezar o custo da energia.
\end{abstract}

Palavras-chave: evapotranspiração, microaspersão, modelo de otimização, custos, hidráulica

\section{Microirrigation system design through non linear programming considering different water demands}

\begin{abstract}
In this work the optimum microsprinkler system design is analyzed through optimization model using non linear programming, with the objective of minimizing operational and investment costs, for three sceneries of the water demand in two localities of the Northeast region of Brazil: Iguatu, Ceará State, with annual and monthly water balances, and João Pessoa, Paraíba State, with monthly water balance. The model has as decision variables: the lengths of the pipe sections with their respective diameters, the daily irrigation total time, the emission uniformity, the irrigation frequency and the emitter mean operation pressure. The results evidenced that in semi-arid climate areas, as Iguatu, there are not important differences in the design system with the demands obtained through the annual or monthly water balances. The investment cost was not affected by the demands, but just the operational and total system costs. Regions with smaller daily and annual water requirement, as João Pessoa, allow larger number of operational units of the system and, therefore, larger reduction of the investment cost. However, for same number of the subunit and operational units the water demands differences do not affect the design. The maximum daily water demand of the crop has larger effect on the hydraulic system than the annual water demand. The system with optimum design depends on the combined analysis of the subunit number and of operational units of the system and the energy cost should not be ignored.
\end{abstract}

Key words: evapotranspiration, microsprinkler, optimization model, costs, hydraulic

${ }^{1}$ CEFET-PB. Av. $1^{\circ}$ de Maio, João Pessoa, PB, CEP 58015 430. Fone: (83) 3208 3073. E-mail: kennedyflavio@yahoo.com.br 2 DEC/UFCG. Av. Aprígio Veloso. Campina Grande, PB, CEP 58109 970. Fone: (83) 3310 1155. E-mail: carlos-galvao@uol.com.br ${ }^{3}$ DTCC/UFPB. Cidade Universitária. João Pessoa, PB, CEP 58050 900. Fone: (83) 3216 7684. E-mail: heberp@uol.com.br 


\section{INTRODUÇÃO}

A adoção dos sistemas de microirrigação tem tido crescente evolução no Brasil, mesmo em áreas com poucos problemas de escassez hídrica, para uma grande variedade de culturas e climas. Aliados à característica de maior potencial de economia de água, esses sistemas proporcionam também melhor controle das operações e maior economia de mão-de-obra e energia; no entanto, os custos de investimento desses sistemas continuam relativamente elevados em função, principalmente, da necessidade de muitos equipamentos.

Diversos estudos têm sido desenvolvidos nos últimos anos com o objetivo de aperfeiçoar o dimensionamento dos sistemas de microirrigação e difundir novas aplicações de técnicas matemáticas e computacionais. Embora a hidráulica desses sistemas esteja bem compreendida, muitos trabalhos têm, como foco principal, este aspecto, entre os quais: Hathoot \& Al-Amoud (1993), Kang \& Nishiyama (1996a,b), Anwar (1999a,b e 2000), Vallesquino \& Luque-Escamilla (2001) e Valiantzas (2002). Outros trabalhos enfocam a importância da análise econômica dos investimentos, com dois principais objetivos: (a) a minimização dos custos de investimento e operacionais (b) maximização dos benefícios. Dentre essas análises destacam-se os trabalhos de Holzapfel et al. (1990), Dandy \& Hassanli (1996), Saad \& Frizzone (1996) e Saad \& Mariño (2002).

Alguns dos fatores que afetam o dimensionamento hidráulico e os custos dos sistemas de microirrigação, são: o formato e a topografia da área, o layout do sistema, as características do emissor, o número de subunidades, a estratégia de operação das subunidades e o plano tarifário de energia. Considerando-se áreas com diferentes climas, além desses fatores, a demanda hídrica da cultura também pode afetar significativamente o dimensionamento.

Muitos estudos têm enfatizado a determinação das necessidades hídricas da planta, que são fundamentais para o dimensionamento e a operação dos sistemas de irrigação e para o manejo da cultura; além disso, afetam os custos da irrigação e podem ser um fator decisivo na escolha do local em que se implantará um projeto, mas poucos estudos têm considerado a influência da demanda hídrica no dimensionamento do sistema. É comum não se considerar a contribuição das precipitações e se dimensionar o sistema com base na máxima evapotranspiração da cultura (Marouelli, 1983; Saad, 1990; Back, 1997). Alguns autores, porém, recomendam que as precipitações sejam consideradas com certo nível de probabilidade de ocorrência na elaboração dos projetos, apesar das incertezas (Silva et al., 1988; Bernardo, 1989). Matos (2000) verificou que a evapotranspiração anual foi o fator com maior efeito nos custos do sistema de microaspersão por unidade de área, dentre outras variáveis analisadas.

Rotineiramente, os sistemas de microirrigação são dimensionados com o objetivo de reduzir o custo inicial, desprezando a influência do custo da energia, mas esta estratégia, além de não garantir a obtenção da melhor concepção de projeto, pode implicar em desvantagens competitivas no mercado, em função do elevado consumo de energia, inviabili- zando o empreendimento. Outro aspecto relevante para o dimensionamento é a necessidade que se apresenta de projetos cada vez mais racionais, não apenas economicamente mas, também, sob o ponto de vista energético. Nos sistemas de microirrigação o consumo da energia está relacionado à topologia da rede de irrigação e à estratégia de operação adotada, sobretudo com a demanda hídrica da cultura; já o custo com a energia depende do seu consumo e da tarifa contratada.

Este trabalho apresenta o desenvolvimento de um modelo computacional de programação não linear para a análise do dimensionamento de sistemas de microirrigação com layout quadrado ou retangular e com desníveis uniformes, considerando-se o efeito de diferentes demandas hídricas nos custos de investimento e energia, para várias configurações e operações de sistemas.

\section{MATERIAL E MÉTODOS}

\section{Construção do modelo}

Função objetivo: O modelo de otimização tem como função objetivo a minimização dos custos totais do sistema de microirrigação, compostos neste estudo pelos custos de investimento e custos operacionais:

$$
\operatorname{Min} f(x)=C_{T}=\frac{C_{I}+C_{O P}}{A_{I}}
$$

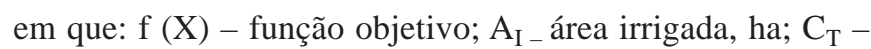
custo total, US\$ ano ${ }^{-1}$ ha-1 $^{-1} \mathrm{C}_{\mathrm{I}}$ - custo de investimento amortizado, US\$ ano-1; e $\mathrm{C}_{\mathrm{OP}}$ - custo operacional, US\$ ano-1. Equivalência entre moedas: US\$ 1,00 = R\$ 3,0.

Cálculo dos custos de investimento: Os custos de investimento amortizados $\left(\mathrm{C}_{\mathrm{I}}\right)$ foram calculados segundo Lucena (2003) e compreendem: (1) tubulações de polietileno $\left(\mathrm{C}_{\mathrm{PE}}\right)$ e de PVC ( $\left.\mathrm{C}_{\text {PVC }}\right)$, função do diâmetro e classe de pressão; (2) conjunto de sucção $\left(\mathrm{C}_{\mathrm{SUC}}\right)$, função do diâmetro; (3) sistema de bombeamento $\left(\mathrm{C}_{\mathrm{SB}}\right)$, função da potência consumida; (4) sistema de filtragem $\left(\mathrm{C}_{\mathrm{SF}}\right)$, função da vazão total do sistema; (5) centro de controle $\left(\mathrm{C}_{\mathrm{CC}}\right)$ e válvulas $\left(\mathrm{C}_{\mathrm{VR}}\right)$, função do número de subunidades; (6) adaptadores de linhas laterais $\left(\mathrm{C}_{\mathrm{AD}}\right)$ e conectores $\left(\mathrm{C}_{\mathrm{CON}}\right)$, função do número de linhas laterais; (7) emissores $\left(\mathrm{C}_{\mathrm{EM}}\right)$; e (8) medidor de energia $\left(\mathrm{C}_{\mathrm{MEN}}\right)$.

Cálculo do custo operacional: Neste trabalho, os custos operacionais são representados apenas pelos custos com energia elétrica consumida pelo bombeamento anual para o consumidor do Grupo B (baixa tensão), Subgrupo B2 Rural, onde não se inclui o custo de demanda (ANEEL, 2000). Para esta classe de consumidor é possível desconto na tarifa em função do horário de operação, mas neste estudo foi utilizada a tarifa sem desconto.

Consideraram-se duas formas de cálculo do custo operacional, em função da determinação de requerimento hídrico anual para o projeto: a) o custo operacional com a demanda hídrica obtida pelo balanço hídrico anual e b) o custo operacional com a demanda hídrica obtida com o balanço hídrico mensal. 


$$
\begin{gathered}
\mathrm{C}_{\mathrm{OPa}}=\frac{\mathrm{C}_{\mathrm{EN}} \mathrm{T}_{\mathrm{TID}} \text { NDIa } \mathrm{P}_{\mathrm{OT}}}{\mathrm{I}_{\mathrm{F}}} \\
\mathrm{C}_{\mathrm{OPm}}=\frac{\sum_{\mathrm{j}=1}^{12}\left(\mathrm{C}_{\mathrm{EN}} \mathrm{T}_{\mathrm{TID}_{\mathrm{j}}} \mathrm{NDIm}_{\mathrm{j}}\right) \mathrm{P}_{\mathrm{OT}}}{\mathrm{I}_{\mathrm{F}}}
\end{gathered}
$$

em que: $\mathrm{C}_{\mathrm{OPa}}$ - custo operacional, considerando-se o balanço hídrico anual, US\$ ano-1; $\mathrm{C}_{\mathrm{OPm}}$ - custo operacional, tendo em vista o balanço hídrico mensal, US\$ ano ${ }^{-1}$; NDIa número de dias irrigados ao ano; NDIm $_{j}$ - número de dias do mês ${ }_{j} ; C_{E N}$ - custo da tarifa de energia elétrica, US\$ kW h ${ }^{-1} ; \mathrm{T}_{\mathrm{TID}}$ - tempo total diário de irrigação com balanço hídrico anual ou com balanço hídrico mensal (índice ${ }_{j}$ ), h; $I_{F}$ - freqüência de irrigação, dia; e $P_{O T}$ - potência requerida pelo sistema de bombeamento, $\mathrm{kW}$.

$$
\begin{gathered}
\mathrm{T}_{\mathrm{TID}}=\mathrm{T}_{\text {IUOmed }} \mathrm{N}_{\mathrm{UO}} \\
\mathrm{T}_{\mathrm{IUO}} \mathrm{med}_{\mathrm{med}}=\frac{\mathrm{Vol}_{\text {med }}}{\mathrm{q}_{\mathrm{e}} \mathrm{N}_{\mathrm{EP}}} \\
\mathrm{Vol}_{\text {med }}=\frac{\mathrm{R}_{\mathrm{HC} \text { med }} \mathrm{E}_{\mathrm{E}} \mathrm{E}_{\mathrm{L}} \mathrm{I}_{\mathrm{F}}}{\mathrm{Ea}} \\
\mathrm{q}_{\mathrm{e}}=\mathrm{k}_{\mathrm{d}} \mathrm{h}^{\mathrm{x}}
\end{gathered}
$$

em que: $\mathrm{T}_{\text {IUOmed }}$ - tempo de irrigação médio por unidade operacional, h; $\mathrm{Vol}_{\text {med }}$ - demanda média bruta diária d’água da cultura, L; $\mathrm{N}_{\mathrm{UO}}$ - número de unidades operacionais; $\mathrm{q}_{\mathrm{e}-}$ vazão do emissor, $\mathrm{L} \mathrm{h}^{-1}$; $\mathrm{E}_{\mathrm{E}}$ e $\mathrm{E}_{\mathrm{L}}$ - respectivamente espaçamento entre plantas na fileira e entre fileiras, $m, I_{F}-$ freqüência de irrigação, dia; $\mathrm{R}_{\mathrm{HCmed}}$ - requerimento hídrico médio da cultura, mm d ${ }^{-1} ; \mathrm{N}_{\mathrm{EP}}$ - número de emissores por planta e Ea - eficiência de aplicação d’água, decimal; h - pressão de serviço do emissor, $\mathrm{m}$; $\mathrm{k}_{\mathrm{d}}$ coeficiente de descarga do emissor; e $\mathrm{x}$ - expoente que caracteriza o regime de fluxo do emissor.

\section{Variáveis de decisão do modelo}

As variáveis de decisão do modelo estão definidas com base na configuração e operação do sistema, isto é, em função do número de subunidades e unidades operacionais, e com base no cálculo da demanda hídrica. Tem-se, como variáveis de decisão, os comprimentos dos trechos de tubos com seus respectivos diâmetros, o tempo total diário de irrigação, a uniformidade de aplicação d’água, a freqüência de irrigação e a pressão média de operação do emissor. O número de subunidades e unidades operacionais é definido previamente, sendo, portanto, conhecidos os comprimentos das tubulações.

A topologia da rede hidráulica compreende uma linha principal, linhas secundárias, linhas de derivação e linhas laterais. A linha principal, com apenas um trecho, e as linhas secundárias podem ter até cinco segmentos, com diâmetros diferentes em cada trecho de tubulação. As linhas de derivação e linhas laterais têm dois trechos com diâmetros diferentes, enquanto os comprimentos dos trechos de laterais são função do número de emissores e os trechos da derivação são função do número de saídas de laterais.
O número de trechos das linhas secundárias varia de acordo com o número total de subunidades do sistema; assim, quanto maior o número de subunidades, maior também o número de trechos da secundária e o número de variáveis.

Cálculo da perda de carga nas tubulações: As perdas de carga nas linhas laterais são determinadas pela equação de Darcy-Weisbach, com o fator de atrito f de Blasius e nas tubulações que não são de polietileno (sucção, principal, secundária e de derivação) as perdas de carga são determinadas pela equação de Hazen-Williams.

\section{Restrições do modelo}

O modelo apresenta restrições lineares e não-lineares, e de contorno para variáveis, tendo-se:

- Pressão de operação do emissor

$$
\mathrm{h}_{\min } \leq \mathrm{h} \leq \mathrm{h}_{\max }
$$

em que: $h=h_{\text {med }}$ - pressão média ou de serviço do emissor na subunidade, $\mathrm{m} ; \mathrm{h}_{\min }-$ mínima pressão de operação do emissor, m; e $h_{\max }$ - máxima pressão de operação do emissor, m.

- Variação de pressão na subunidade

Combinando-se as equações da uniformidade de emissão (UE) de Karmeli \& Keller (1975), com a recomendação de Keller \& Bliesner (1990) para máxima variação de pressão na subunidade na fase de projeto, tem-se a perda de carga admissível $\left(\Delta \mathrm{h}_{\mathrm{S}}\right)$ :

$$
\Delta \mathrm{h}_{\mathrm{S}} \leq 2,5 \mathrm{~h}\left\{1-\left[\left(\frac{\mathrm{UE}}{100\left(1-1,27 \mathrm{C}_{\mathrm{VF}} \mathrm{N}_{\mathrm{EP}}{ }^{-0,5}\right)}\right)\right]^{\frac{1}{\mathrm{x}}}\right\}
$$

em que: $\Delta \mathrm{h}_{\mathrm{s}}$ - perda de carga admissível na subunidade m; $\mathrm{UE}$ - uniformidade na emissão; $\mathrm{C}_{\mathrm{VF}}$ - coeficiente de variação de fabricação do emissor, decimal; $\mathrm{x}$ - expoente da equação característica do emissor; $\mathrm{N}_{\mathrm{EP}}$ - número de emissores por planta; e h - pressão de operação do emissor, m.

- Distribuição da perda de carga na subunidade

$$
0 \leq \mathrm{h}_{\mathrm{fL}}+\mathrm{h}_{\mathrm{fD}} \leq \Delta \mathrm{h}_{\mathrm{S}}
$$

em que: $h_{\mathrm{fL}}$ e $\mathrm{h}_{\mathrm{fD}}$ - perdas da carga nas linhas laterais e de derivação, $\mathrm{m}$, respectivamente.

- Comprimento da linha lateral

$$
\left(\mathrm{N}_{\mathrm{ei}}+\mathrm{N}_{\mathrm{ej}}\right) \mathrm{S}_{\mathrm{e}}=\mathrm{L}_{\mathrm{L}}
$$

em que: $\mathrm{N}_{\mathrm{ei}}$ e $\mathrm{N}_{\mathrm{ej}}$ - número de emissores no primeiro e segundo trecho da lateral, respectivamente; $\mathrm{S}_{\mathrm{e}}$ - espaçamento entre emissores na lateral, $\mathrm{m}$; e $\mathrm{L}_{\mathrm{L}}$ - comprimento da lateral, $\mathrm{m}$.

- Comprimento da linha de derivação

$$
\left(\mathrm{N}_{\mathrm{Si}}+\mathrm{N}_{\mathrm{Sj}}\right) \mathrm{S}_{\mathrm{L}}=\mathrm{L}_{\mathrm{D}}
$$

em que: $\mathrm{N}_{\mathrm{Si}}$ e $\mathrm{N}_{\mathrm{Sj}}$ - número de saídas de laterais no primeiro e segundo trecho de um lado da derivação, respectivamente; $\mathrm{S}_{\mathrm{L}}$ - espaçamento entre laterais na derivação, m; e $\mathrm{L}_{\mathrm{D}}$ comprimento da derivação, m. 
- Comprimento da linha secundária

$$
\begin{gathered}
\mathrm{L}_{\mathrm{S}}=\sum \mathrm{L}_{\mathrm{Sij}}=\frac{\mathrm{L}_{\mathrm{X}}}{2}-\mathrm{L}_{\mathrm{L}} \\
\mathrm{L}_{\mathrm{S}_{\mathrm{ij}}}=\mathrm{L}_{\mathrm{L}}, \text { para } \mathrm{j}=1 \\
\mathrm{~L}_{\mathrm{Sij}}=2 \mathrm{~L}_{\mathrm{L}}, \text { para } \mathrm{j} \neq 1
\end{gathered}
$$

em que: $\mathrm{L}_{\mathrm{S}}$ - comprimento da linha secundária, $\mathrm{m}$; $\mathrm{L}_{\mathrm{Sij}}-$ comprimento do segmento i do trecho j da linha secundária, $\mathrm{m}$; e $\mathrm{L}_{\mathrm{X}}$ - comprimento da área irrigada, $\mathrm{m}$.

- Comprimento da linha principal

$$
\mathrm{L}_{\mathrm{P}}=\sum \mathrm{L}_{\mathrm{Pi}}=\frac{\mathrm{L}_{\mathrm{Y}}}{2}
$$

em que: $L_{P}$ - comprimento da linha principal, $m$; $L_{P i}-$ comprimento do trecho i da principal, m; e $\mathrm{L}_{\mathrm{Y}}$ - largura da área irrigada, m.

- Velocidades nas tubulações $\left(\mathrm{m} \mathrm{s}^{-1}\right)$, por trecho ou por segmento de: lateral $\left(\mathrm{V}_{\mathrm{L}}\right)$, derivação $\left(\mathrm{V}_{\mathrm{D}}\right)$, secundária $\left(\mathrm{V}_{\mathrm{S}}\right)$ e principal $\left(\mathrm{V}_{\mathrm{P}}\right)$

$$
\mathrm{V}_{\mathrm{L}}, \mathrm{V}_{\mathrm{D}}, \mathrm{V}_{\mathrm{S}} \text { e } \mathrm{V}_{\mathrm{P}} \leq 2,5
$$

- Tempo máximo de operação diária

$$
\begin{gathered}
\mathrm{N}_{\text {UOmax }} \mathrm{T}_{\text {IUOmax }} \leq \mathrm{T}_{\mathrm{ID}} \\
\mathrm{T}_{\mathrm{IUO}_{\max }}=\frac{\mathrm{Vol}_{\text {max }}}{\mathrm{q}_{\mathrm{e}} \mathrm{N}_{\mathrm{EP}}} \\
\mathrm{Vol}_{\text {max }}=\frac{\mathrm{R}_{\mathrm{HC}} \mathrm{max}_{\mathrm{E}} \cdot \mathrm{E}_{\mathrm{E}} \cdot \mathrm{E}_{\mathrm{L}} \cdot \mathrm{I}_{\mathrm{F}}}{\mathrm{Ea}}
\end{gathered}
$$

em que: $\mathrm{N}_{\mathrm{UOmax}}$ - número máximo de unidades operacionais; $\mathrm{T}_{\text {IUOmax }}$ - tempo de irrigação máximo por unidade operacional, h; T ID - tempo disponível de irrigação diária, h; $\mathrm{Vol}_{\max }$ - máxima demanda bruta diária d’água da cultura, L e $\mathrm{R}_{\mathrm{HCmax}}$ - requerimento hídrico máximo da cultura, $\mathrm{mm} \mathrm{d}^{-1}$.

- Máxima precipitação do emissor

$$
\mathrm{P}_{\mathrm{m}} \leq \mathrm{I}_{\mathrm{S}}
$$

$$
\mathrm{P}_{\mathrm{m}}=\frac{4 \mathrm{q}_{\mathrm{e}}}{\pi \mathrm{D}_{\mathrm{m}}^{2}}
$$

em que: $\mathrm{P}_{\mathrm{m}}$ - precipitação do emissor, $\mathrm{mm} \mathrm{h}^{-1}$; $\mathrm{q}_{\mathrm{e}}$ - vazão do emissor, $\mathrm{L} \mathrm{h}^{-1} ; \mathrm{D}_{\mathrm{m}}$ - diâmetro molhado do emissor, m; e $\mathrm{I}_{\mathrm{s}}$ - máxima capacidade de infiltração d’água no solo, $\mathrm{mm} \mathrm{h}^{-1}$.

- Máxima área molhada pelo emissor

$$
A_{M}=\frac{0,785 D_{m}^{2}}{E_{E} E_{L}} 100 \leq A_{M_{\max }}
$$

em que: $A_{M}$ - área molhada pelo emissor, \% e $A_{M}$ - máxima área molhada permissível, \%.

- Máxima lâmina armazenável no solo

$$
\mathrm{L}_{\mathrm{M}}=\frac{\mathrm{R}_{\mathrm{HC}_{\max }} \mathrm{I}_{\mathrm{F}}}{\mathrm{Ea}} \leq \frac{\mathrm{L}_{\mathrm{Mmax}} \mathrm{A}_{\mathrm{M}}}{100}
$$

em que: $\mathrm{L}_{\mathrm{M}}$ - lâmina armazenada no solo, mm e $\mathrm{L}_{\mathrm{Mmax}}-$ máxima lâmina armazenável no solo, mm.

- Outras restrições numéricas às variáveis de decisão

$$
\begin{gathered}
\mathrm{N}_{\mathrm{ei}}, \mathrm{N}_{\mathrm{ej}}, \mathrm{N}_{\mathrm{Si}}, \mathrm{N}_{\mathrm{Sj}} \geq 1 \text { (int eiro) } \\
\mathrm{UE}_{\min } \leq \mathrm{UE} \leq \mathrm{UE}_{\max } \\
\mathrm{I}_{\mathrm{Fmin}_{\min }} \leq \mathrm{I}_{\mathrm{F}} \leq \mathrm{I}_{\mathrm{Fmax}_{\max }}
\end{gathered}
$$

\section{Técnica de otimização}

O problema é do tipo programação não linear restrita

\begin{tabular}{|c|c|c|c|c|c|c|c|c|c|c|c|}
\hline $\mathrm{N}_{\text {SUT }}$ & $A_{l}$ (ha) & $\mathrm{L}_{X} / \mathrm{L}_{Y}$ & $L_{L}(m)$ & $L_{D}(m)$ & $L_{s}(m)$ & $L_{P}(m)$ & & & $N_{U 0}$ & & \\
\hline 4 & 33,8688 & 0,98 & 144 & 294 & 144 & 294 & 1 & 2 & 4 & 8 & - \\
\hline 8 & 33,8688 & 0,98 & 72 & 294 & 216 & 294 & 1 & 2 & 4 & 8 & - \\
\hline 12 & 33,8688 & 0,98 & 48 & 294 & 240 & 294 & 1 & 2 & 3 & 6 & 12 \\
\hline 16 & 34,6752 & 1,30 & 42 & 258 & 294 & 258 & 1 & 2 & 4 & 8 & 16 \\
\hline 20 & 34,2720 & 2,06 & 42 & 204 & 378 & 204 & 1 & 2 & 5 & 10 & 20 \\
\hline
\end{tabular}
e é solucionado utilizando-se o algoritmo Programação Quadrática Seqüencial (SQP) do software Matlab versão 5.3 .

Dados para aplicação do modelo: Esta análise foi realizada para diferentes configurações e operações de sistemas de microaspersão. As dimensões dos sistemas foram preestabelecidas e definidas em função do espaçamento entre emissores e se analisaram várias possibilidades de projeto, variando o número de subunidades e de unidades operacionais (Tabela 1 ).

Os sistemas foram dimensionamentos para demandas obtidas a partir do balanço hídrico anual em Iguatu, CE, e do balanço hídrico mensal para as localidades de Iguatu e João

Tabela 1. Configurações e operações de sistemas analisados

$\mathrm{N}_{\mathrm{SUT}}$ - Número total de subunidades; $\mathrm{N}_{\mathrm{UO}}$ - Número de unidades operacionais; $\mathrm{A}_{1}$ - Área irrigada, ha 
Tabela 2. Requerimentos hídricos diários médios e máximos nas localidades de Iguatu,CE, e João Pessoa, PB, obtidos a partir do balanço hídrico mensal (Lucena, 2003)

\begin{tabular}{|c|c|c|c|c|c|c|c|c|c|c|c|c|}
\hline \multicolumn{13}{|c|}{ Requerimentos hídricos em Iguatu, CE, mm d-1 } \\
\hline Mês & Jan & Fev & Mar & Abr & Mai & Jun & Jul & Ago & Set & Out & Nov & Dez \\
\hline $\mathrm{R}_{\mathrm{HC} \text { méd }}$ & 2,8 & 1,2 & 0,1 & 0,3 & 2,0 & 2,4 & 2,5 & 2,9 & 3,3 & 3,5 & 3,5 & 3,3 \\
\hline $\mathrm{R}_{\text {HC Max }}$ & 3,4 & 1,4 & 0,1 & 0,4 & 2,4 & 2,9 & 3,0 & 3,5 & 4,0 & 4,2 & 4,2 & 4,0 \\
\hline \multicolumn{13}{|c|}{ Requerimentos hídricos em João Pessoa, $\mathrm{PB}, \mathrm{mm} \mathrm{d}^{-1}$} \\
\hline $\mathrm{R}_{\text {HC med }}$ & 2,1 & 1,8 & 0,1 & 0,0 & 0,0 & 0,0 & 0,0 & 0,0 & 0,4 & 1,8 & 2,3 & 2,5 \\
\hline $\mathrm{R}_{\text {HC max }}$ & 2,5 & 2,2 & 0,1 & 0,0 & 0,0 & 0,0 & 0,0 & 0,0 & 0,5 & 2,2 & 2,8 & 3,0 \\
\hline
\end{tabular}

Tabela 3. Relação de diâmetros comerciais aplicáveis em cada uma das tubulações da rede e seus respectivos custos

\begin{tabular}{|c|c|c|c|c|c|c|c|c|c|c|c|}
\hline \multicolumn{3}{|c|}{ Principal ( $\left.L_{P}\right)$} & \multicolumn{3}{|c|}{ Secundária $\left(L_{S}\right)$} & \multicolumn{3}{|c|}{ Derivação $\left(L_{D}\right)$} & \multicolumn{3}{|c|}{ Lateral $\left(\mathrm{L}_{\mathrm{L}}\right)$} \\
\hline $\begin{array}{l}\mathrm{DN} \\
(\mathrm{mm})\end{array}$ & $\begin{array}{c}\text { DI } \\
(\mathrm{mm})\end{array}$ & $\begin{array}{l}\text { Custo } \\
\left.\text { (US\$ } \mathrm{m}^{-1}\right)\end{array}$ & $\begin{array}{l}\text { DN } \\
(\mathrm{mm})\end{array}$ & $\begin{array}{c}\text { DI } \\
(\mathrm{mm})\end{array}$ & $\begin{array}{c}\text { Custo } \\
\left.\text { (US\$ } \mathrm{m}^{-1}\right)\end{array}$ & $\begin{array}{c}\text { DN } \\
(\mathrm{mm})\end{array}$ & $\begin{array}{c}\text { DI } \\
(\mathrm{mm})\end{array}$ & $\begin{array}{c}\text { Custo } \\
\text { (US\$ } \mathrm{m}^{-1} \text { ) }\end{array}$ & $\begin{array}{l}\text { DN } \\
(\mathrm{mm})\end{array}$ & $\begin{array}{c}\text { DI } \\
(\mathrm{mm})\end{array}$ & $\begin{array}{c}\text { Custo } \\
\text { (US } \$ \mathrm{~m}^{-1} \text { ) }\end{array}$ \\
\hline 50 & 48,05 & 0,81 & 50 & 48,05 & 0,81 & 50 & 48,05 & 0,81 & 12 & 9,95 & 0,167 \\
\hline 75 & 72,45 & 1,55 & 75 & 72,45 & 1,55 & 75 & 72,45 & 1,55 & 16 & 13,2 & 0,223 \\
\hline 100 & 97,45 & 2,47 & 100 & 97,45 & 2,47 & 100 & 97,45 & 2,47 & 20 & 17,0 & 0,303 \\
\hline 125 & 119,75 & 3,67 & 125 & 119,75 & 3,67 & 125 & 119,75 & 3,67 & 25 & 21,2 & 0,367 \\
\hline 150 & 143,70 & 4,43 & 150 & 143,70 & 4,43 & 150 & 143,70 & 4,43 & 30 & 27,2 & 0,480 \\
\hline $200^{*}$ & 212,45 & 7,17 & 200 & 212,45 & 7,17 & 200 & 212,45 & 7,17 & - & - & - \\
\hline 250 & 262,35 & 9,13 & 250 & 262,35 & 9,13 & - & - & - & - & - & - \\
\hline 300 & 312,20 & 11,07 & 300 & 312,20 & 11,07 & - & - & - & - & - & - \\
\hline
\end{tabular}

* Para DN $\geq 200 \mathrm{~mm}$, pressão nominal (PN) de $60 \mathrm{~m}(600 \mathrm{kPa})$, para as demais PN de $40 \mathrm{~m}(400 \mathrm{kPa})$. Com m = m.c.a.

Pessoa, PB, tomando-se como base os resultados de Lucena (2003). Nas Tabelas 2, 3 e 4 são apresentados os principais dados de entrada do modelo.

Tabela 4. Informações para entrada no modelo

\begin{tabular}{|c|c|}
\hline Cultura selecionada & $\begin{array}{c}\text { Manga } \\
\text { (Mangifera indica L.) }\end{array}$ \\
\hline $\begin{array}{l}\text { Capacidade de infiltração máxima d'água no solo }\left(\mathrm{I}_{\mathrm{S}}\right) \text {, } \\
\mathrm{mm} \mathrm{h}^{-1}\end{array}$ & 15 \\
\hline Coeficiente de variação de fabricação do emissor $\left(\mathrm{C}_{\mathrm{VF}}\right)$ & 0,04 \\
\hline $\begin{array}{l}\text { Coeficiente de rugosidade da Equação de Hazen- } \\
\text { Williams }(C)\end{array}$ & 145 \\
\hline Custo da tarifa de energia elétrica $\left(C_{E N}\right)$, US $\$ \mathrm{~kW} \mathrm{h-1}$ & 0,11554 \\
\hline Desnível do terreno, $\mathrm{m}$ & 0 \\
\hline Diâmetro molhado pelo emissor, $\mathrm{m}$ & $\mathrm{D}_{\mathrm{m}}=0,9198 \mathrm{~h}^{0,5435}$ \\
\hline Eficiência de aplicação (Ea),decimal & 0,90 \\
\hline Equação característica do emissor, $\mathrm{L} \mathrm{h}^{-1}$ & $\mathrm{q}_{\mathrm{e}}=19,342 \mathrm{~h}^{0,3775}$ \\
\hline Espaçamento da cultura, m (EMBRAPA, 2002) & $6 \times 6$ \\
\hline Espaçamento entre emissores na lateral $\left(\mathrm{S}_{\mathrm{e}}\right), \mathrm{m}$ & 6 \\
\hline Espaçamento entre saídas na derivação $\left(\mathrm{S}_{\mathrm{L}}\right), \mathrm{m}$ & 6 \\
\hline Faixa de pressões de operação do emissor, m & $15 \leq h \leq 30$ \\
\hline Fator de Recuperação do Capital (FRC) & 0,10296 \\
\hline Freqüência de irrigação $\left(\mathrm{I}_{\mathrm{F}}\right)$, dia & $1 \leq I_{F} \leq 5$ \\
\hline Máxima lâmina armazenável no solo $\left(\mathrm{L}_{\mathrm{M}}\right), \mathrm{mm}$ & 100 \\
\hline $\begin{array}{l}\text { Máxima área molhada }\left(A_{M \max }\right) \text {, \% (Keller \& Bliesner, } \\
\text { 1990) }\end{array}$ & 67 \\
\hline Número de emissores por planta $\left(\mathrm{N}_{\mathrm{EP}}\right)$ & 1 \\
\hline $\begin{array}{l}\text { Número de dias irrigados ao ano (NDla) (Lucena, } \\
\text { 2003) }\end{array}$ & 174,4 \\
\hline $\begin{array}{l}\text { Requerimento hídrico médio da cultura em Iguatu } \\
\left(\mathrm{R}_{\mathrm{HCmed}}\right), \mathrm{mm} \mathrm{d}^{-1} \text { (Lucena, 2003) }\end{array}$ & 2,96 \\
\hline $\begin{array}{l}\text { Requerimento hídrico máximo da cultura em Iguatu } \\
\left(\mathrm{R}_{\mathrm{HC} \text { max }}\right), \mathrm{mm} \mathrm{d}^{-1} \text { (Lucena, 2003) }\end{array}$ & 4,20 \\
\hline Tempo total diário de operação disponível (T $\left.T_{I D}\right), h$ & 21 \\
\hline Uniformidade de emissão (UE), \% & $90 \leq \mathrm{UE} \leq 100$ \\
\hline
\end{tabular}

\section{RESULTADOS E DISCUSSÃO}

\section{Análise dos custos}

Custo de investimento: Em função do máximo requerimento hídrico diário da cultura $\left(\mathrm{R}_{\mathrm{HCmax}}\right)$ (Tabela 2$)$, das características hidráulicas do emissor, da freqüência de irrigação ótima de 1 dia e da restrição de tempo diário de operação disponível, em Iguatu, só são permitidas no máximo oito unidades operacionais $\left(\mathrm{N}_{\mathrm{UOmax}}=8\right)(\mathrm{Eq}$. 18) para os balanços hídricos anual e mensal, mas em João Pessoa é possível o emprego de até doze unidades operacionais $\left(\mathrm{N}_{\mathrm{UOmax}}=12\right)$.

Pode-se verificar, na Tabela 5, que os custos de investimento são decrescentes do maior número de subunidades ( $\left.\mathrm{N}_{\mathrm{SUT}}\right)$, até certo limite, após o qual passam a ser crescentes porque, à medida que o tamanho das subunidades diminui, os diâmetros mínimos comerciais são atingidos, fazendo com que o maior número de acessórios, como válvulas e conectores de linhas, eleve os custos do sistema. Variações nos custos de investimento de até 10,3\% são observadas para um mesmo número de unidades operacionais $\left(\mathrm{N}_{\mathrm{UO}}\right)$. $\mathrm{O}$ aumento no número de unidades operacionais também diminui acentuadamente os custos de investimento, conseqüência direta da redução de vazão conduzida no sistema, implicando em: 1) menores diâmetros das tubulações, que atendam às restrições impostas e, portanto, menores custos; 2) menor investimento em alguns componentes do sistema, cujos custos são determinados diretamente pela vazão aduzida, a exemplo dos sistemas de bombeamento e de tratamento d'água; logo, o menor custo de investimento é obtido, em todas as configurações, com o maior $\mathrm{N}_{\mathrm{Uo}}$ possível, o que está de acordo com a recomendação de Keller \& Bliesner (1990). Desta forma, o maior custo de investimento do sistema é obtido 
para $\mathrm{N}_{\mathrm{UO}}=1$, em todas as configurações analisadas, e o menor custo de investimento em Iguatu é obtido com $\mathrm{N}_{\mathrm{UO}}=8$ $\left(\mathrm{N}_{\mathrm{SUT}}=8\right.$ e 16), enquanto em João Pessoa o menor custo de investimento é obtido com $\mathrm{N}_{\mathrm{UO}}=10\left(\mathrm{~N}_{\mathrm{SUT}}=20\right)$ (Tabela 5).

As demandas hídricas obtidas com os balanços hídricos anual ou mensal promovem alterações desprezíveis nos custos de investimento, comparando-se a mesma operação, ou seja, mesmo $\mathrm{N}_{\mathrm{UO}}$, em todas as configurações analisadas ( $\left.\mathrm{N}_{\text {SUT }}\right)$. Entre os diferentes balanços e mesma operação, a máxima variação entre os custos de investimento não supera 2,90\%, significando que a demanda total anual de irrigação tem influência irrelevante nos custos de investimento, sendo o dimensionamento baseado principalmente em critérios e restrições hidráulicas estabelecidos. Na Figura 1 apresentam-se os custos de investimento médios otimizados, em função do $\mathrm{N}_{\mathrm{UO}}$, para o sistema com doze subunidades que pode representar o comportamento dos custos das demais configurações.

As variáveis climáticas interferem no dimensionamento da rede, mas apenas quando a máxima demanda hídrica diária da cultura afeta o número máximo de unidades operacionais. Em João Pessoa, onde a demanda hídrica diária máxima é inferior à de Iguatu, são possíveis as operações com $\mathrm{N}_{\mathrm{UO}}=10$ e 12, promovendo importante redução nos custos de investimento.

Em média, o custo de investimento máximo nos três requerimentos hídricos calculados é de US\$ ha-1 $\mathrm{ano}^{-1} 232,60$ $\left(\mathrm{N}_{\mathrm{SUT}}=4\right.$ e $\left.\mathrm{N}_{\mathrm{UO}}=1\right)$ e o mínimo de US\$ ha-1 ano $^{-1} 150,80$ $\left(\mathrm{N}_{\mathrm{SUT}}=20\right.$ e $\left.\mathrm{N}_{\mathrm{UO}}=10\right)$, uma variação de $54,2 \%$. O sistema com doze subunidades possibilita o máximo número de unidades operacionais $\left(\mathrm{N}_{\mathrm{UO}}=12\right)$, o que poderia levar à máxima redução nos custos de investimento em função da vazão

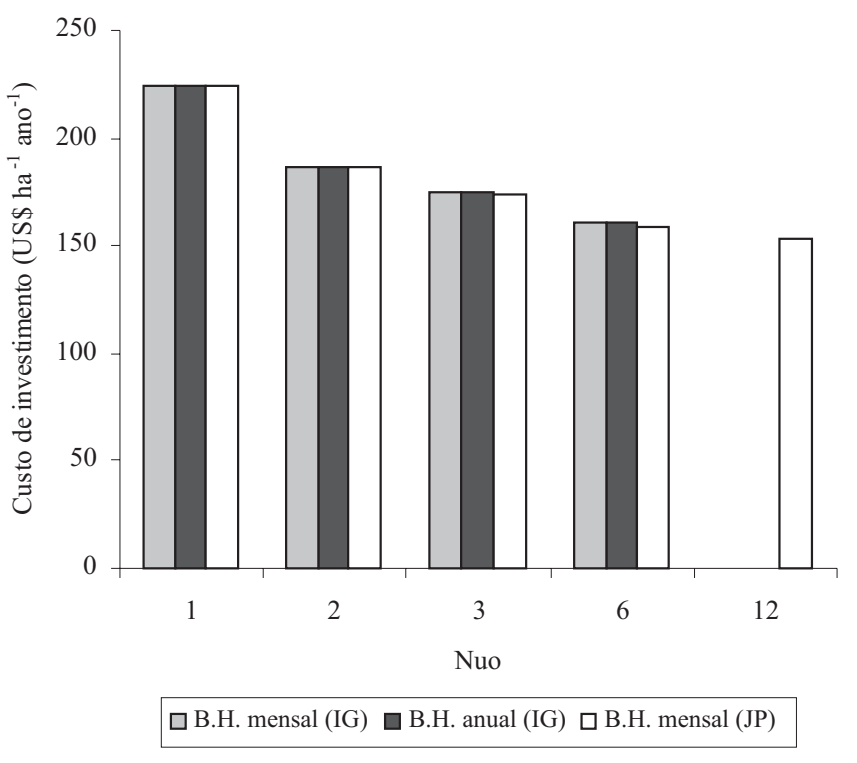

Figura 1. Variação dos custos de investimento com as demandas hídricas, para o sistema com doze subunidades $\left(N_{S U T}=12\right)$, em função do número de unidades operacionais $\left(\mathrm{N}_{\mathrm{UO}}\right)$

bombeada. No entanto, o dimensionamento com $\mathrm{N}_{\mathrm{SUT}}=20$ e $\mathrm{N}_{\mathrm{UO}}=10$ resulta em menores custos de investimento pela influência do menor custo da subunidade, embora com menor número de unidades operacionais.

Custo operacional: As demandas hídricas anuais obtidas nos diferentes balanços são crescentes para João Pessoa (mensal), Iguatu (anual) e Iguatu (mensal), implicando em tempos totais de irrigação também crescentes. Deste modo, os custos operacionais (Eqs. 2 e 3) são maiores com o balanço hídrico

Tabela 5. Custos otimizados (US\$ ha $a^{-1}$ ano-1) de investimento $\left(C_{1}\right)$, operacionais $\left(C_{O P}\right)$ e totais $\left(C_{T}\right)$ do sistema, para as diferentes demandas hídricas, configurações e operações

\begin{tabular}{|c|c|c|c|c|c|c|c|c|c|c|c|}
\hline \multirow{2}{*}{$A_{1}$} & \multirow{2}{*}{$\mathrm{N}_{\text {SUT }}$} & \multirow{2}{*}{$\mathrm{N}_{\mathrm{UO}}$} & \multicolumn{3}{|c|}{ B. H. mensal (IG) } & \multicolumn{3}{|c|}{ B. H. mensal (JP) } & \multicolumn{3}{|c|}{ B. H. anual (IG) } \\
\hline & & & $C_{I}$ & $\mathrm{C}_{\mathrm{OP}}$ & $\mathrm{C}_{\mathrm{T}}$ & $C_{I}$ & $\mathrm{C}_{\mathrm{OP}}$ & $\mathbf{C}_{T}$ & $C_{I}$ & $\mathrm{C}_{\mathrm{OP}}$ & $\mathrm{C}_{\mathrm{T}}$ \\
\hline \multirow{3}{*}{33,8688} & \multirow{3}{*}{4} & 1 & 233,20 & 51,10 & 284,30 & 232,70 & 20,00 & 252,70 & 232,00 & 31,10 & 263,10 \\
\hline & & 2 & 197,10 & 56,90 & 254,00 & 197,10 & 22,30 & 219,40 & 199,00 & 34,20 & 233,20 \\
\hline & & 4 & 178,10 & 52,90 & 231,00 & 180,20 & 20,50 & 200,70 & 178,60 & 32,10 & 210,70 \\
\hline \multirow{4}{*}{33,8688} & \multirow{4}{*}{8} & 1 & 223,80 & 50,40 & 274,20 & 224,60 & 19,90 & 244,40 & 223,80 & 30,70 & 254,50 \\
\hline & & 2 & 187,20 & 55,60 & 242,70 & 187,00 & 22,30 & 209,30 & 187,20 & 33,80 & 221,00 \\
\hline & & 4 & 168,50 & 48,50 & 217,10 & 168,40 & 19,30 & 187,70 & 169,20 & 30,10 & 199,30 \\
\hline & & 8 & 159,90 & 66,60 & 226,50 & 155,40 & 21,20 & 176,60 & 159,70 & 40,00 & 199,80 \\
\hline \multirow{5}{*}{33,8688} & \multirow{5}{*}{12} & 1 & 224,40 & 52,10 & 276,50 & 224,30 & 24,40 & 248,70 & 223,70 & 30,50 & 254,30 \\
\hline & & 2 & 186,60 & 55,60 & 242,20 & 186,50 & 22,50 & 209,00 & 186,50 & 33,80 & 220,40 \\
\hline & & 3 & 174,40 & 50,90 & 225,30 & 174,30 & 20,80 & 195,10 & 174,60 & 30,80 & 205,30 \\
\hline & & 6 & 161,00 & 46,80 & 207,80 & 159,30 & 19,90 & 179,20 & 160,90 & 28,60 & 189,50 \\
\hline & & 12 & - & - & - & 153,10 & 30,80 & 183,90 & - & - & - \\
\hline \multirow{4}{*}{34,6752} & \multirow{4}{*}{16} & 1 & 219,10 & 51,10 & 270,20 & 218,60 & 21,10 & 239,70 & 220,20 & 30,80 & 251,00 \\
\hline & & 2 & 182,60 & 56,40 & 239,00 & 182,40 & 22,70 & 205,10 & 183,70 & 33,70 & 217,40 \\
\hline & & 4 & 163,80 & 49,60 & 213,40 & 163,40 & 20,50 & 184,00 & 163,70 & 30,50 & 194,10 \\
\hline & & 8 & 156,00 & 64,90 & 220,90 & 152,00 & 19,90 & 171,90 & 154,60 & 40,80 & 195,40 \\
\hline \multirow{4}{*}{34,2720} & \multirow{4}{*}{20} & 1 & 222,80 & 53,10 & 275,90 & 223,50 & 20,40 & 243,90 & 222,00 & 31,90 & 253,90 \\
\hline & & 2 & 185,20 & 65,50 & 250,80 & 186,30 & 23,00 & 209,30 & 183,40 & 36,10 & 219,50 \\
\hline & & 5 & 160,90 & 50,00 & 210,90 & 158,80 & 20,60 & 179,40 & 162,70 & 32,30 & 195,00 \\
\hline & & 10 & - & - & - & 150,80 & 29,40 & 180,20 & - & - & - \\
\hline
\end{tabular}

B. H. anual (IG) - balanço hídrico anual em Iguatu; B. H. mensal (IG) - balanço hídrico mensal em Iguatu; B. H. mensal (JP) - balanço hídrico mensal em João Pessoa 
mensal em Iguatu e menores em João Pessoa para a mesma operação ( $\left.\mathrm{N}_{\mathrm{UO}}\right)$. Espera-se que os custos operacionais cresçam com o maior $\mathrm{N}_{\mathrm{UO}}$, porém existem operações em que a redução da potência do sistema permite que o tempo de irrigação seja aumentado em função do $\mathrm{N}_{\mathrm{UO}}$, sem elevar os custos com energia, o que pode ser constatado na Figura 2.

Comparando-se os custos operacionais em Iguatu, verifica-se que o balanço hídrico mensal resulta em aumentos que variam entre 54,8 e 81,4\% com relação ao balanço anual; já com relação a João Pessoa, o balanço hídrico mensal em Iguatu promove aumentos entre 113,8 e 226,4\% (Tabela 5).

Os custos operacionais representaram entre 18,0 e 29,4\% dos custos totais com o balanço hídrico mensal em Iguatu, e entre 11,8 e 20,9\% para o balanço anual. Em João Pessoa representaram entre 7,9 e 16,7\%, considerando-se todas as configurações $\left(\mathrm{N}_{\mathrm{SUT}}\right)$ e operações $\left(\mathrm{N}_{\mathrm{UO}}\right)$. Deste modo, considerando-se as amplas faixas de variações dos custos operacionais para cada caso analisado, constata-se a importância de uma avaliação minuciosa das opções de projeto, tendo em vista a relevância que apresentam esses custos em toda a vida útil do projeto de irrigação.

Custo total: Observou-se que os custos de investimento compreenderam a maior porcentagem dos custos totais do sistema de microaspersão, da mesma forma que os custos de investimento são reduzidos com maior $\mathrm{N}_{\mathrm{UO}}$; os custos totais também tendem a reduzir, mas a definição do sistema com custo mínimo depende dos custos operacionais; assim, os maiores custos totais são obtidos em todas as configurações $\left(\mathrm{N}_{\mathrm{SUT}}\right)$ com uma unidade operacional $\left(\mathrm{N}_{\mathrm{UO}}=1\right)$ e os de menor custo total variam com $\mathrm{N}_{\mathrm{SUT}}$ e $\mathrm{N}_{\mathrm{UO}}$ (Tabela 5); logo, não se deve dimensionar o sistema considerando-se apenas os custos de investimento, devendo-se analisar as possíveis combinações de configurações $\left(\mathrm{N}_{\mathrm{SUT}}\right)$ e operações $\left(\mathrm{N}_{\mathrm{UO}}\right)$ para se obter o sistema de custo mínimo. Na Figura 3 tem-se a

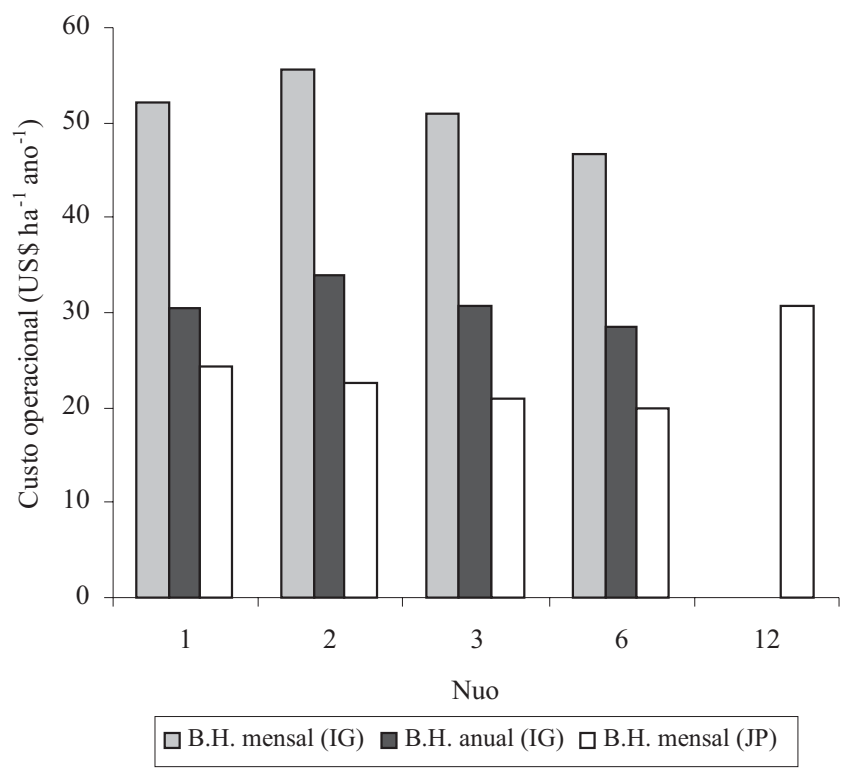

Figura 2. Variação dos custos operacionais com as demandas hídricas, para o sistema com doze subunidades $\left(N_{S U T}=12\right)$, em função do número de unidades operacionais $\left(\mathrm{N}_{\cup \mathrm{O}}\right)$

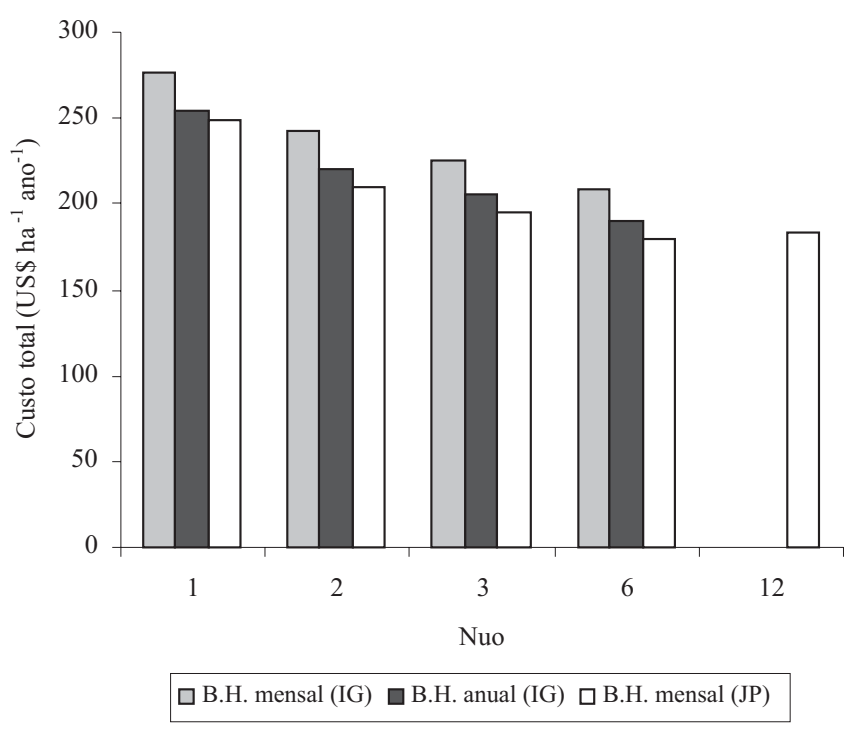

Figura 3. Variação dos custos totais com as demandas hídricas para o sistema com doze subunidades $\left(\mathrm{N}_{\mathrm{SUT}}=12\right)$, em função do número de unidades operacionais $\left(\mathrm{N}_{\cup \mathrm{O}}\right)$

variação dos custos totais com as demandas hídricas para o sistema com doze subunidades.

Para a localidade de Iguatu as configurações e operações de sistemas que resultam nos custos totais mínimos não diferem para os balanços hídricos anual e mensal, ou seja, $\mathrm{N}_{\mathrm{SUT}}=12$ e $\mathrm{N}_{\mathrm{UO}}=6$. Embora tenha ocorrido aumento de $9,6 \%$ nos custos totais com o balanço hídrico mensal, isto retrata a influência da demanda hídrica anual, que afeta o tempo total de irrigação, com conseqüências nos custos operacionais; já o custo total mínimo do sistema em João Pessoa é obtido com $\mathrm{N}_{\mathrm{SUT}}=16$ e $\mathrm{N}_{\mathrm{UO}}=8$. Entre os custos totais mínimos ótimos, obtidos com os balanços hídricos anual e mensal, em Iguatu, e o custo mínimo em João Pessoa, verificam-se diferenças de 10,2 e 20,9\%, respectivamente. Esses resultados revelam a importância das demandas hídricas na elaboração do projeto de irrigação e, conseqüentemente, no planejamento dos investimentos.

\section{Análise hidráulica}

Hidraulicamente não há diferenças relevantes no dimensionamento da rede, considerando-se as diferenças de demandas hídricas com os balanços em Iguatu (anual e mensal) e João Pessoa (mensal), quando a mesma pressão de serviço (h) é otimizada para determinada operação. Além dos custos de investimento refletirem este comportamento, outros indicadores permitem tal interpretação sobre a hidráulica da rede (Tabela 6). Observa-se, por exemplo, que para um mesmo número de unidades operacionais praticamente não há diferença na potência requerida $\left(\mathrm{P}_{\mathrm{OT}}\right)$ do sistema de bombeamento.

Em todas as configurações $\left(\mathrm{N}_{\mathrm{SUT}}\right)$ e operações com $\mathrm{N}_{\mathrm{UO}}<8$, o modelo busca, sempre, a pressão de operação mínima do emissor de $15,0 \mathrm{~m}$ e a uniformidade de emissão (UE) mínima de 90\%. Porém, quando o sistema é dimensionado com oito ou mais unidades operacionais $\left(\mathrm{N}_{\mathrm{UO}} \geq 8\right)$ é necessária a elevação da pressão de serviço para 
Tabela 6. Pressão de serviço $(\mathrm{m})$, uniformidade de emissão (\%), variação de pressão na subunidade $(\mathrm{m})$ e potência requerida (kW), em função de $\mathrm{N}_{\text {SUT, }}, \mathrm{N}_{\mathrm{UO}}$ e das demandas hídricas

\begin{tabular}{|c|c|c|c|c|c|c|c|c|c|c|c|c|c|}
\hline \multirow{2}{*}{$\mathrm{N}_{\text {SUT }}$} & \multirow{2}{*}{$\mathrm{N}_{\mathrm{UO}}$} & \multicolumn{4}{|c|}{ B. H. mensal (IG) } & \multicolumn{4}{|c|}{ B. H. mensal (JP) } & \multicolumn{4}{|c|}{ B. H. anual (IG) } \\
\hline & & h & UE & $\Delta \mathrm{h}_{\mathrm{sr}}$ & $P_{0 T}$ & h & UE & $\Delta \mathbf{h}_{\mathrm{sr}}$ & $P_{0 T}$ & h & UE & $\Delta \mathrm{h}_{\mathrm{sr}}$ & $P_{\text {0т }}$ \\
\hline \multirow{3}{*}{4} & 1 & 15,0 & 90,0 & 4,93 & 70,8 & 15,0 & 90,0 & 4,98 & 70,9 & 15,0 & 90,0 & 5,00 & 71,0 \\
\hline & 2 & 15,0 & 90,0 & 5,01 & 39,5 & 15,0 & 90,0 & 5,01 & 36,5 & 15,0 & 90,0 & 4,42 & 39,1 \\
\hline & 4 & 15,0 & 90,0 & 4,98 & 18,3 & 15,0 & 90,0 & 4,45 & 18,2 & 15,0 & 90,0 & 4,97 & 18,3 \\
\hline \multirow{4}{*}{8} & 1 & 15,0 & 90,0 & 5,10 & 70,1 & 15,0 & 90,0 & 4,78 & 70,2 & 15,0 & 90,0 & 5,10 & 70,1 \\
\hline & 2 & 15,0 & 90,0 & 5,10 & 38,6 & 15,0 & 90,0 & 5,10 & 36,4 & 15,0 & 90,0 & 5,10 & 38,6 \\
\hline & 4 & 15,0 & 90,0 & 5,10 & 16,9 & 15,0 & 90,0 & 5,10 & 17,1 & 15,0 & 90,0 & 4,86 & 17,2 \\
\hline & 8 & 24,7 & 90,0 & 8,14 & 14,0 & 15,0 & 90,0 & 5,10 & 9,4 & 24,2 & 90,0 & 8,04 & 13,7 \\
\hline \multirow{5}{*}{12} & 1 & 15,0 & 90,0 & 4,98 & 72,5 & 15,3 & 90,0 & 5,04 & 72,3 & 15,0 & 90,0 & 4,98 & 69,7 \\
\hline & 2 & 15,0 & 90,0 & 4,98 & 38,6 & 15,0 & 90,0 & 4,93 & 39,9 & 15,0 & 90,0 & 4,98 & 38,6 \\
\hline & 3 & 15,0 & 90,0 & 4,98 & 23,6 & 15,0 & 90,0 & 4,93 & 24,6 & 15,0 & 90,0 & 4,98 & 23,4 \\
\hline & 6 & 15,0 & 90,0 & 4,98 & 10,8 & 15,0 & 90,0 & 4,93 & 11,8 & 15,0 & 90,0 & 4,98 & 10,9 \\
\hline & 12 & - & - & - & - & 28,6 & 90,0 & 9,39 & 11,6 & - & - & - & - \\
\hline \multirow{4}{*}{16} & 1 & 15,0 & 90,0 & 5,30 & 72,8 & 15,0 & 90,0 & 5,30 & 76,4 & 15,0 & 90,0 & 4,65 & 72,1 \\
\hline & 2 & 15,0 & 90,0 & 5,17 & 40,1 & 15,0 & 90,0 & 5,20 & 41,2 & 15,0 & 90,0 & 4,65 & 39,4 \\
\hline & 4 & 15,0 & 90,0 & 5,30 & 17,7 & 15,0 & 90,0 & 5,17 & 18,6 & 15,0 & 90,0 & 5,30 & 17,8 \\
\hline & 8 & 24,7 & 90,0 & 8,04 & 13,9 & 15,0 & 90,0 & 4,65 & 9,0 & 24,2 & 90,0 & 7,94 & 14,3 \\
\hline \multirow{4}{*}{20} & 1 & 15,0 & 90,0 & 4,65 & 74,7 & 15,0 & 90,0 & 3,50 & 73,0 & 15,0 & 90,0 & 4,04 & 73,7 \\
\hline & 2 & 15,0 & 90,0 & 4,65 & 46,1 & 15,0 & 90,0 & 3,48 & 41,2 & 15,0 & 90,0 & 4,69 & 41,8 \\
\hline & 5 & 15,0 & 90,0 & 5,03 & 14,1 & 15,0 & 90,0 & 5,30 & 14,8 & 15,0 & 90,0 & 3,60 & 14,9 \\
\hline & 10 & - & - & - & - & 26,9 & 90,0 & 9,17 & 13,1 & - & - & - & - \\
\hline
\end{tabular}

o atendimento do limite de tempo de operação diário disponível, a exemplo de $\mathrm{N}_{\mathrm{UO}}=10$ e 12 , para o caso do balanço hídrico em João Pessoa. Esta informação revela que o dimensionamento e a operação que resultam em menores custos totais para o sistema requerem o uso das menores pressões de serviço e menores uniformidades de emissão admissíveis. Observa-se, também, que o modelo não eleva a pressão de serviço para que ocorra a redução dos custos com tubulações, pois isto implicaria em aumento do custo energético e de alguns componentes do sistema. A redução de potência com o aumento do número de unidades operacionais ocorre principalmente pela redução da vazão bombeada e não pela redução da altura manométrica; trata-se de importante informação para o dimensionamento, em particular no que diz respeito à seleção do emissor.

\section{CONCLUSÕES}

1. O modelo de programação não linear desenvolvido pode ser aplicado com adequação para análise e dimensionamento de sistema de microirrigação.

2. O sistema de microirrigação com maior número de subunidades e maior o número de unidades operacionais, até certo limite máximo, reduz os custos de investimento.

3. A obtenção do sistema de custo ótimo depende da análise conjunta do número de subunidades e unidades operacionais.

4. Em regiões semi-áridas, as necessidades hídricas diárias e anuais da cultura, determinadas através dos balanços hídricos anual ou mensal, promovem diferenças em termos de custos operacionais, mas não afetam o dimensionamento ótimo do sistema.
5. A máxima demanda hídrica diária da cultura exerce maior efeito no dimensionamento do sistema do que a demanda hídrica anual.

\section{LITERATURA CITADA}

ANEEL - Agência Nacional de Energia Elétrica. Resolução n. 456. 2000. 53p.

Anwar, A. A. Factor G for pipelines with equally spaced multiple outlets and outflow. Journal of Irrigation and Drainage Engineering, New York, v.125, n.1, p.34-38, 1999a.

Anwar, A. A. Adjusted factor Ga for pipelines with multiple outlets and outflow. Journal of Irrigation and Drainage Engineering, New York, v.125, n.6, p.355-359, 1999b.

Anwar, A. A. Inlet pressure for horizontal tapered laterals. Journal of Irrigation and Drainage Engineering, New York, v.126, n.1, p.57-63, 2000.

Back, A. J. Determinação da precipitação efetiva para irrigação suplementar pelo balanço hídrico horário: Um caso-estudo em Urussanga. Porto Alegre: UFRGS, 1997. 132p. Tese Doutorado

Bernardo, S. Manual de irrigação. 5.ed. Viçosa: Imprensa Universitária, 1989. 586p.

Dandy, G. C.; Hassanli, A. M. Optimum design and operation of multiple subunit drip irrigation systems. Journal of Irrigation and Drainage Engineering, New York, v.122, n.5, p.265275. 1996.

EMBRAPA - Empresa Brasileira de Pesquisa Agropecuária. Manga. http://www.cnpmf.embrapa.br/manga.html. 12 Ago. 2002.

Karmeli, D.; Keller, J. Trickle irrigation design. Rain bird sprinkler manufacturing corporation. California: Glendora, 1975. 133p.

Keller, J.; Bliesner, R. D. Sprinkle and trickle irrigation. New York: Van Nostrand Reinhold, 1990. 652p. 
Hathoot, H. M.; Al-Amoud, A. I. Analysis and design of trickle irrigation laterals. Journal of Irrigation and Drainage Engineering, New York, v.119, n.5, p.756-767, 1993.

Holzapfel, E. A.; Mariño, M. A.; Valenzuela, A. Drip irrigation nonlinear optimization model. Journal of Irrigation and Drainage Engineering, New York, v.116, n.4, p. 479-496, 1990.

Kang, Y.; Nishiyama, S. Analysis and design of microirrigation laterals. Journal of Irrigation and Drainage Engineering, New York, v.122, n.2, p.75-82, 1996a.

Kang, Y.; Nishiyama S. Design of microirrigation submain units. Journal of Irrigation and Drainage Engineering, New York, v. 122, n. 2, p. 83-89, 1996 b.

Lucena, K. F. M. Modelo de otimização para o dimensionamento e operação de sistemas de microirrigação. Campina Grande: UFPB. 2003. 187p. Tese Doutorado

Marouelli, W. A. Análise de distribuição das probabilidades de chuva visando ao manejo da irrigação suplementar. Viçosa: UFV. 1983. 127p. Dissertação Mestrado

Matos, J. A. Aplicação da programação não linear no dimensionamento de sistemas de irrigação localizada. Botucatu: UNESP. 2000. 89p. Tese Doutorado
Saad, J. C. C. Estudo da distribuição de freqüência da evapotranspiração e da precipitação pluvial para fins de dimensionamento de sistemas de irrigação. Piracicaba: ESALQ/USP. 1990. 124p. Dissertação Mestrado

Saad, J. C. C.; Frizzone, J. A. Design and management optimization of trickle irrigation systems using non-linear programming. Journal of Agricultural Engineering Research. Silsoe Research Institute, Silsoe, UK, v.64, p.109-118, 1996.

Saad, J. C. C.; Mariño, M. A. Modelos de programação linear e não linear para otimização do dimensionamento de sistemas de irrigação localizada. In: Congresso Brasileiro de Engenharia Agrícola, 31, 2002. Salvador. Anais... Salvador: SBEA, 2002, p.978-981,

Silva, W. L. C.; Oliveira, C. A. S.; Marouelli, W. A. Subsídios para dimensionamento de sistemas de irrigação. In: Congresso Nacional de Irrigação e Drenagem, 8, 1988. Florianópolis. Anais... Florianópolis. ABID. 1988, p.535-553.

Valiantzas, J. D. Continuous outflow variation along irrigation laterals: Effect of the number of outlets. Journal of Irrigation and Drainage Engineering, New York, v. 128, n.1, p.34-42, 2002.

Vallesquino, P.; Luque-escamilla, P. L. New algorithm for hydraulic calculation in irrigation laterals. Journal of Irrigation and Drainage Engineering, New York, v. 127, n.4, p.254-260, 2001. 